\title{
PÍO DEL RÍO HORTEGA EN MÉXICO Y CUBA. 1930
}

\author{
Juan del Río-Hortega Bereciartu \\ Doctor en Medicina. Profesor Asociado de Historia de la Medicina. Universidad de Valladolid.
}

ABSTRACT The article informs about of invitation mades by doctor Gutiérrez Perrin to Pio del Rio Hortega to travel to México and deliver a course of thirteen lessons. After their trip to México, Rio Hortega goes to La Habana and deliver a serial of conferences.

KEY WORDS México. Cuba. Pío del Río Hortega biography. 1930.
RESUMEN El artículo narra la invitación realizada a Pío del Río Hortega por el doctor Gutiérrez Perrín para viajar a México e impartir allí un ciclo de trece lecciones. Tras el viaje a México, Río Hortega se traslada a La Habana donde imparte otro ciclo de conferencias.

PALABRAS CLAVE México. Cuba. Pío del Río Hortega, biografía. 1930.
El 18 de Febrero de 1930, Don José Castillejo y Duarte, Secretario de la Junta para Ampliación de Estudios e Investigaciones Científicas, escribía a Río Hortega ${ }^{1}$ : «Mi querido amigo: el Sr. Menéndez Pidal ha recibido anoche un cablegrama desde Méjico que dice: "esperamos entusiásticamente Río. Perrínn. Este cablegrama es contestación a uno enviado al Sr. Perrín diciéndole que usted podria ir en el mes de Mayo.... Esta invitación no debió sorprender a Don Pío, ya que en 1929 Don Tomás Gutiérrez Perrín, su fraternal amigo y condiscípulo desde las enseñanazas de López García en Valladolid y posteriormente Catedrático de Fisiología de la Facultad de Medicina y de la Escuela Médico-Militar de México, visitó en Madrid su laboratorio de la Residencia de Estudiantes y comentaron la posibilidad de realizar este viaje. Pero sin duda, Río Hortega pensaría que las fechas elegidas para cruzar el Atlántico no eran las más oportunas: Max Borst, el Director del Instituto de Patologia de Munich, esperaba que Don Pio diera un ciclo de conferencias en Alemania precisamente en la primavera ${ }^{2}$-viaje que en un principio se aplazó a Noviembre de 1930 y que finalmente se realizó en Enero de 1931-. Lhermitte, Crouzon y Roussy, Presidente, Secretario General y Vicepresidente, respectivamente, de la Sociedad de Neurología de París, le hablan invitado a dar una conferencia sobre la microglía en la cátedra de tan ilustre institución en Abrilı ${ }^{3}$, pero tuvieron que conformarse con leer el trabajo que Don Pío les envió por correo; y finalmente, el Presidente del Centro de Neurologia de Bruselas, el Doctor Laurelle, esperaba que
Don Pío acudiera a su aula para hablar sobre las lesiones elementales de los centros nerviosos cuando hubiera finalizado su trabajo en Paris ${ }^{4}$, esperanza que obviamente se vio frustrada.

Una vez organizado el viaje a México, Don Pío pensó que podría aprovechar la travesía atlántica para visitar los Estados Unidos y Canadá, accediendo así a los deseos que Penfield, tan reiterativamente, le reivindicaba. Así se lo cuenta a su amigo Camilo Trefogli, Catedrático de Histologia de la Facultad de Veterinaria de la Universidad de Buenos Aires, en una carta ${ }^{5}$ fechada el 13 de Mayo: "...En estos días estoy sumamente atareado preparando un nuevo viaje a América, pero esta vez a México y Estados Unidos. Tengo el propósito de marchar el $1^{\circ}$ de Junio para regresar a principios de Septiembre....n. Pero, tanto la prolongada estancia en México, como la inesperada invitación de la Sociedad Hispano-Cubana de Cultura para dar un curso de conferencias en La Habana, truncó, una vez más, su viaje a los territorios de Penfield.

Río Hortega embarca en el puerto de Santander el 30 de Mayo de 1930; asi se lo relata a su familia ${ }^{6}$ : «...esta tarde a las 5 embarcaré pero solo por un momento, pues el barco no sale hasta medianoche. Ayer, entre lluvia incesante, estuvimos en Santillana. Hoy comeremos con los de Valdecilla...."

Don Pío llegó a México a mediados de Junio de 1930 -habiendo arribado previamente algún día en La Habana- 
invitado por el Instituto Hispano-Mexicano de Intercambio Universitario, cuyo Presidente era Don Alejandro Quijano y vicepresidente Don Tomás Gutiérrez Perrin; sin embargo, el Decano de la Facultad de Medicina, Doctor Fernando Ocaranza, y el Director del Instituto de Biologia de México, Doctor Isaac Ochotorena, participaron muy activamente para que el éxito de la misión cientifica fuera definitivo.

Antes de comenzar el curso de conferencias y lecciones prácticas que se le había encomendado, el día 23 de Junio la Universidad Nacional de México rindió un multitudinario homenaje al cientifico español. El acto tuvo lugar a las 12 del mediodía en el Paraninfo de la Universidad, y el Rector, Don Ignacio Garcia Téllez, le otorgó el diploma y la medalla que le acreditaban el título de Profesor Extraordinario de la Universidad Nacional de México ${ }^{7}$. Presidieron el evento, el Rector, Perrín, Quijano, el Embajador de España -Vizconde de Gracia Real- y Don Pío, quien presentó el programa que iba a seguir durante su magisterio mexicano. Así pues, según reza el programa ${ }^{8}$ del IX Curso del Instituto Hispano-Mexicano de Intercambio Universitario, Río Hortega va a realizar un curso de laboratorio y un curso de conferencias. El primero " ... Consistirá en la aplicación de las técnicas propias del Doctor Río Hortega al estudio de la histología normal y patológica, en general, y especialmente del sistema nervioso. Dichas técnicas se referirán a tinciones nucleares, protoplásmicas, del tejido conjuntivo, del sistema retículoendotelial, de la microglia, de la oligodendroglia y de las células nerviosas. Los asistentes [cuyo número se ha limitado a 15] podrán estudiar el material que prefieran: tejidos, órganos, tumores, etc., guiados por el Doctor Río Hortega, quien les orientará en la lectura e interpretación de las preparaciones. Los trabajos tendrán lugar diariamente, de 11 a 2, en el laboratorio de Fisiología de la Facultad de Medicinan. En cuanto al curso de conferencias "....Constará de un ciclo de 13 lecciones sobre citología normal y patológica del sistema nervioso central que serán explicadas con proyecciones los martes y sábados, en el Salón de Actos de la Facultad de Medicina, según el orden siguiente:

\section{Constitución histológica de la glándula pineal} (26 Junio).

Estructura normal y patológica de la célula nerviosa (28 Junio).
Morfología y junción de la oligodendroglia (5 Julio). Alteraciones generales de la neuroglía (8 Julio). Las gliosis (12 Julio). Los gliomas (15 Julio). La microglía en reposo (19 Julio).

La jagocitosis en los centros nerviosos (22 Julio). Los procesos inflamatorios de los centros nerviosos (26 Julio).

La necrobiosis de los centros nerviosos (27 Julio). La reparación de las heridas cerebrales (2 de Agosto).

Los endoteliomas meníngeos (5 Agosto).

\section{LA ASISTENCIA AL CURSO DE CONFERENCIAS ES LIBRE}

Pues bien, tras la presentación de este apretadísimo programa, Don Pío se dispone a impartir su esperado curso ante un variopinto alumnado formado por Catedráticos, profesores y estudiantes de la Facultad de Medicina. Entre los asistentes, destaca la presencia de Perrin, Ocaranza, Ochotorena, Manuel Martínez Baez -Catedrático de HistologiaIgnacio Chávez e Ignacio González Guzmán; estos últimos serian, años más tarde, íntimos colaboradores de Isaac Costero ${ }^{9}$.

Pero Don Pío no se limitaría a completar este intenso curso; los científicos mexicanos quisieron aprovechar al máximo la estancia del histólogo español y "exprimir» el fruto de sus enseñanzas. De esta manera, La Sociedad Mexicana de Biología requirió, los días 21 y 22 de Julio la presencia de RíoHortega presentar un trabajo ${ }^{10}$ en la XVII Reunión de la misma. En la recepción de Don Pío ante los miembros de la institución mexicana, habló el profesor Martínez Báez, quien en un extensísimo y elogioso discurso ${ }^{11}$ dijo entre otras cosas: «....Hay en la visita que hoy hace el doctor Río Hortega, a nuestro pais, un detalle, que nosotros tenemos el deber de conocer y de apreciar y sobre el cual no quiero pasar en silencio. Para realizar su viaje a México, tuvo el Doctor Río Hortega que declinar la invitación que recibió para exponer sus descubrimientos, durante las vacaciones de este año, en algunas universidades alemanas, así como la apremiente solicitud de Roussy, el ilustre Profesor de Anatomía Patológica de la Universidad de Paris, que, en nombre de los histopatólogos franceses, urgía a Río Hortega para que se presentase a leer su comunicación ante el Congreso Internacional de Neurología, reunido en Paris. Y es que el Doctor Río Hortega, noblemente humano, como un verda-

ARBOR CLXXXI 714 JULIO-AGOSTO (2005) 207-211 ISSN: 0210-1963 
dero sabio moderno, dejó el alago de los sabios europeos para cumplir un deber de patriotismo, en la más amplia y elevada de sus aceptaciones...."

El día 22 de Julio, el Presidente de la Academia Nacional de Medicina de México, el Doctor José Torres Torija, escribia ${ }^{12}$ a Don Pío para solicitar su presencia en el acto que, en su honor, se iba a celebrar a las 7 de la tarde del día siguiente en la ilustre casa, y donde Río Hortega expondría, una vez más, otra conferencia. La presentación de Don Pío fue obra del Doctor Arroyo, quien elogió ampliamente la personalidad del portillano.

Antes de embarcar hacia Cuba, el Instituto Hispano-Mexicano de Intercambio Universitario dio un multitudinario banquete-homenaje en honor de Río Hortega que se celebró en el restaurante San Angel Inn, el 5 de Agosto ${ }^{13}$.

De la estancia de Don Pio en La Habana tenemos menos información. Sabemos que, como ya hemos comentado, no entraba en los planes del histólogo la visita a la isla antillana; de hecho, escribe a su padre ${ }^{14}$ desde México: "Queridisimo padre: supongo seguirá cuidándose mucho. Ya tengo muchisimas ganas de verle. Tengo todo arreglado para salir de aquí el 8 de Agosto y llegar ahí hacia el 26..... Es, precisamente, al final de su estancia en México, cuando recibe una carta de su antiguo discipulo desde el magisterio que ejerció en Buenos Aires en 1925, el neurocirujano cubano Carlos M. Ramirez Corría, quien le dice lo siguiente ${ }^{15}$ : "...Como le ofrecimos, ibamos a hacer algo en su contra cuando Ud. pasara de nuevo por aqui de regreso a su patria "viejan. Y cuando pensamos traicionarle, nunca hubimos de suponer hasta qué grado de importancia iba a resultar grande la mala acción. Pero es el hecho, paradójico en extremo en nuestro medio actual, que la avidez científica criolla no se resigna a verle alejarse sin dejar algo siquiera de aquello a que se refería el verle o y querido maestro de Buenos Aires: le esperamos a Ud. para retenerlo aunque fuera una semana ...... Pero los científicos cubanos, aparte de remitir una invitación firmada por un antiguo discípulo de Rio-Hortega, creyeron conveniente ujugar otra baza", y mandaron a México a un joven médico cubano que, con una carta de Ramirez Corría ${ }^{16}$ y con la mediación de Gutiérrez Perrín, se presentó ante Don Pío. Este joven médico era Don Pedro Manuel León ${ }^{17}$, patólogo del laboratorio del Hospital Municipal de Emergencias "Freyre de Andrade» de la Habana y que, ya de regreso a España, acompañó a Don Pío para aprender sus técnicas en los laboratorios de la Residencia de Estudiantes y del Instituto Nacional del Cáncer.
Don Pío reside aproximadamente veinte dias en La Habana para dar un curso similar -aunque evidentemente menos extenso- que en México. Como ya hemos citado anteriormente, es invitado por la Sociedad Hispano-Cubana de Cultura. El curso tiene lugar en la Facultad de Medicina de la Universidad de La Habana. Entre los asistentes, cabe citar a Pedro Machado, Antonio Navarrete, Angel Vietal Miguel Angel Mendoza, Rodolfo Pérez de los Reyes, Ortelio M. Fortún, René de La Valette, A. Castellanos, Pedro Manuel León. Carlos M. Ramírez Corría, Jaime Cóndom, Pedro Kourí, etc.

El 23 de Agosto, la junta directiva de la Unión Vallisoletana de Cuba, en sesión general extraordinaria presidida por Don Filemón Carmona, le nombra Presidente de Honor de la misma ${ }^{18}$. Don Félix Granados, Presidente del Rotary Club de La Habana, le cursa una invitación para asistir a una sesiónalmuerzo que, en honor del científico español, se celebraría el jueves 21 de Agosto 19 .

El 31 de Agosto, a las 11 de la mañana, Rio Hortega da una conferencia $^{20}$ en el Teatro de La Comedia de La Habana ante los socios de la Sociedad Hispano-Cubana de Cultura. En esta ocasión habló sobre "Algo de Arte Histológico». El 4 de Septiembre, la Facultad de Medicina le rindió un multitudinario banquete-homenaje en el "roof garden" del Hotel Sevilla-Biltmore ${ }^{21}$. El agasajo comenzó a las 9 de la noche y en la presidencia, junto a Don Pío, se sentaron el Rector de la Universidad, Don José Ricardo Martínez, y el Decano de la Facultad de Medicina, Don Luis Felipe Rodríguez Molina. A los postres hablaron el Doctor Ramirez Corría, quien alabó entusiásticamente la obra del sabio español y sus enseñanzas en la ciudad antillana, y el Doctor Rodriguez Molina, quien entre otras cosas dijo ${ }^{22}$ : "... En nuestro deseo de llevar al corazón del doctor del Río Hortega una prueba del gran afecto que le tenemos y en la imposibilidad de rendirle el homenaje más merecido, de haber llegado en periodo de actividades universitarias, de recibirlo en plena sesión del Claustro otorgándole del título de Profesor Honoris Causa, nos limitamos a declararle Profesor de profesores, y le obsequiamos con la medalla del bicentenario de la Universidad de La Habana, para que la conserve como recuerdo de su permanencia entre nosotros, simbolizando el obsequio, la vida e historia de nuestra Universidad creada por los padres dominicos españoles y llevando envuelta en ella la historia de nuestro país..."

El dia 5 de Septiembre fue un dia especialmente agitado para Rio Hortega. A las 12 del mediodia fue homenajeado

ARBOR CLXXXI 714 JULIO-AGOSTO (2005) 207-211 ISSN: 0210-1963 
por sus discípulos en el curso que dio en la cátedra de Histología de la Facultad de Medicina, con un banquete en el Miramar Yacht $\mathrm{Club}^{23}$. Como dice el cronista Miguel Ángel Mendoza: «...Un terceto formado de guitarra, güiro y marimba, sin las horribles e insoportables estridencias de los bongo, amenizó la fiesta, con "sones" y canciones deliciosas escogidas entre lo más selecto de nuestro ritmo criollo, que fueron del agrado del anfitrión..... A las 5 de la tarde presidió la "Sesión fundacional de la Sociedad Cubana de Biologían ${ }^{24}$. El acto tuvo lugar en el salón de actos del Laboratorio de Anatomía Patológica de la Asociación de Dependientes ante una nutrida concurrencia formada por el cuerpo médico de la casa de Salud y numerosos profesionales ajenos a la misma. En la mesa presidencial se sentaron, junto a Río Hortega, el doctor Cárdenas -Director interino del Laboratorio- y los Doctores Ángel Vieta y Ramirez Corría, quien tras presentar elogiosamente a Don Pío dio una conferencia sobre "El efecto de las lecitinas en los órganos de los cobayas normales y tuberculosos». En esta ocasión Río Hortega habló sobre "Aspectos histológicos en la debilidad senil». Por último, a las 9 de la noche, la Academia de Ciencias ofreció a Don Pío una recepción en su honor.

El día 6 de Septiembre a las 11 de la mañana, Don Pío embarcaba en el "Alfonso XIII» con rumbo a España. Como ya hemos comentado iba acompañado por el Doctor Pedro Manuel León, quien residió más de un año en Madrid para aprender las técnicas del maestro español. Tras 5 dias de travesía, el barco llegó al puerto de Nueva York. Don Pío solo tuvo tiempo de enviar un telegrama a sus discipulos norteamericanos más allegados: Penfield, Stevenson y Alpers, quienes días más tarde escribirían a Río Hortega para mostrarle su pena por no haber conseguido retenerle durante algún tiempo en sus universidades 25 .

Para finalizar citaré las palabras que Gutiérrez Perrín escribió a Don Juan del Río Sanz, el padre de Don Pio, tras el regreso de éste a España ${ }^{26}$ : «...Ya está Pío en sus brazos después de haber honrado, una vez más, su limpio apellido. Esta vez llega a España aplaudido por dos naciones que, al admirarle, admiran a nuestra venerada patria. Dios se lo premie..... 
1 Cf: Río-Hortega Bereciartu, J. del. «El Epistolario de Pío del Río-Hortegan, cap. V, doc. 278. Tesis doctoral.

2 Ibidem: Cap. V, docs. 272 y 291.

3 Ibidem: Cap. V, doc. 288.

4 Ibidem:Cap. V, doc. 295.

5 Ibidem: Cap. V, doc. 294.

6 Ibidem:Cap. V, doc. 319.

7 Cf Periódico "Excelsior». "Recepción de un español ilustren. México D.F., martes 24 de Junio de 1930, 1a página. También en: Cf. Periódico "La Presa». "El eminente histólogo Río Hortega en la Universidad". México D.F., martes 24 de Junio de 1930 $1^{\text {a }}$ página.

8 Ibidem: Cap. V. a modo de ilustración.

9 Cf. Costero Tudanca, Isaac. "Crónica de una vocación científican, p. 161. Ed. E. A. México, 1977.

10 Ibidem: Cap. V, doc. 331. Desgraciadamente no hemos conseguido averiguar el trabajo que presentó Río-Hortega ante la Sociedad Mexicana de Biología.

11 Cf. Periódico "El Día Español». "La Sociedad Mexicana de Biología recibió cordialmente al Dr. del Río Hortegan. México D.F. 2 de Agosto de $1930,1^{\text {a }}$ y $4^{\text {a }}$ páginas. La fuente recoge integro el discurso del Dr. Manuel Martínez Báez.

12 Ibidem: Cap. V., doc. 332. Desgraciadamente, no hemos conseguido averiguar el título de la conferencia.

13 lbidem: Cap. V, doc. 335

14 lbidem:Cap. V, doc. 328.

15 Ibidem: Cap. V, doc. 324.

16 Ibidem: Cap. V., doc. 330.

17 Cf: Periódico "El Diario de la Marina», Un patólogo del Hospital Municipal va a España con el Dr. Río Hortegan. La Habana, 6 de Septiembre de 1930.

18 Ibidem: Cap. V., doc. 339. También en: Cano Díaz, Pedro. "Una Contribución a la ciencia histológica: La obra de Don PíoHortega", p. 61. C.S.I.C. Madrid, 1985.

19 Ibidem: Cap. V., doc. 337.
20 Cf: Periódico "El Diario de la Marina». "La Histología en su aspecto científico puro llega a sublimarse y confundirse con la filosofia, dijo ayer en su conferencia el doctor del Río Hortega. La Habana, 1 de Septiembre de 1930. Crónica del Dr. Miguel Angel Mendoza. También en la revista "Surco". "Algo de Arte Histológicor. La Habana, Octubre de 1930. Con posterioridad, D. Pio daria otras conferencias sobre este mismo tema: "Arte y Artificio de la Ciencia Histológican, en el Centro de intercambio Intelectual Germano Español el 14 de Enero de 1932 (/bidem: Cap. VI. docs. 400 y 463. Cap. VII, docs. 468 y 472); publicada en la revista «Residencian, IV (6), 191-206, correspondiente al mes de Diciembre de 1933. Recientemente como facsímil en: López Piñero, José Maria. "Pío del Río-Hortega». Biblioteca de la Ciencia Española I. Fundación Banco Exterior, 405-420. Madrid, 1990. Otra conferencia de similares caracteristicas la dio, ya en el exilio, en el Colegio Libre de Estudios Superiores de Buenos Aires: "Sobre la emoción estética en Histologían (Cf. Rio-Hortega Bereciartu, J. del: "El Magisterio de Pío del Río-Hortega en la Argentina". En: Congreso "Proyección Histórica de España en sus Tres Culturas». Palacio de Las Salinas. Medina del Campo (Valladolid), 15-18 Abril, 1991 (en prensa). Citado también por el Profesor Antonio Orozco Acuaviva en: «El magisterio de Pio del Río Hortega en Hispanoamérican. En las $\mathrm{V}$ Jornadas de Historia de las Ciencias. "Pío del Río-Hortega y la Ciencia de su (1882-1945)". Universidad de Valladolid. 12-14 Noviembre 1991 (en prensa).

21 lbidem: Cap. V, doc. 340.

22 Cf: Periódico "El Diario de La Marina». "La Facultad de Medicina rindió cordial tributo de admiración y afecto al doctor del Rio-Hortegan. La Habana, 5 de Septiembre de 1930. Crónica de Miguel Angel Mendoza.

23 Cf: Periódico "La Voz de Aragón». «Un banquete-homenaje en honor de un profesor español». La Habana, 6 de Septiembre de 1930.
24 Cf: Periódico "El Diario de la Marina». "El doctor Pio del Río Hortega presidió ayer la sesión inaugural de la Sociedad Cubana de Biologian. La Habana, 6 de Septiembre de 1930. Crónica de Miguel Angel Mendoza.

25 Ibidem: Cap. V, docs. 344, 345 y 346.

26 lbidem: Cap. V, doc. 343. 